\title{
Resistance distance in wheels and fans
}

\author{
R. B. Bapat* Somit Gupta ${ }^{\dagger}$
}

February 24, 2009

\begin{abstract}
The wheel graph is the join of a single vertex and a cycle, while the fan graph is the join of a single vertex and a path. The resistance distance between any two vertices of a wheel and a fan are obtained. The resistances are related to Fibonacci numbers and generalized Fibonacci numbers. The derivation is based on evaluating determinants of submatrices of the Laplacian matrix. A combinatorial argument is also illustrated. A connection with the problem of squaring a rectangle is described.
\end{abstract}

\section{Introduction}

We consider graphs which have no loops or parallel edges. Thus a graph $G=$ $(V(G), E(G))$ consists of a finite set of vertices, $V(G)$, and a set of edges, $E(G)$, each of whose elements is a pair of distinct vertices. A weighted graph is a graph in which each edge is assigned a positive number, called its weight. We will assume familiarity with basic graph-theoretic notions, see, for example, Bondy and Murty [4]).

Given a graph, one associates a variety of matrices with the graph. Some of the important ones will be defined now. Let $G$ be a graph with $V(G)=$ $\{1, \ldots, n\}, E(G)=\left\{e_{1}, \ldots, e_{m}\right\}$. The adjacency matrix $A(G)$ of $G$ is an $n \times n$

*Indian Statistical Institute, New Delhi, 110016, rbb@isid.ac.in; The author gratefully acknowledges the support of the JC Bose Fellowship, Department of Science ad Technology, Government of India.

${ }^{\dagger}$ National Institute of Technology Karnataka, Surathkal, Karnataka India, somit.gupta@gmail.com 
matrix with its rows and columns indexed by $V(G)$, and with the $(i, j)$-entry equal to 1 , if vertices $i, j$ are adjacent (i.e., joined by an edge) and 0 otherwise. Thus $A(G)$ is a symmetric matrix with its $i$-th row (or column) sum equal to $d(i)$, which by definition is the degree of the vertex $i, i=1,2, \ldots, n$. Let $D(G)$ denote the $n \times n$ diagonal matrix, whose $i$-th diagonal entry is $d(i), i=$ $1,2, \ldots, n$.

The Laplacian matrix of $G$, denoted by $L(G)$, is defined as $L(G)=D(G)-$ $A(G)$. For a weighted graph $G$, the Laplacian is defined as follows. For $i \neq j$, the $(i, j)$-entry of the Laplacian matrix is set equal to 0 , if $i$ and $j$ are not adjacent, and otherwise it is defined to be the negative inverse (reciprocal) of the weight of the edge $\{i, j\}$. The diagonal entries of the Laplacian matrix are defined so that they make the sum of all entries in their row to be zero.

The resistance distance $r_{i j}$ between vertices $i$ and $j$ in a weighted graph $G$ is defined as the effective electrical resistance between points $i$ and $j$ when we place a resistor along every edge (with the resistance offered by the resistor equal to the weight of the edge) and a potential difference is applied at $i$ and $j$. The resistance matrix $R$ is defined as the matrix with its $(i, j)$-entry equal to $r_{i j}$. There are several equivalent ways to define the resistance distance. We describe some such definitions which will be used. For proofs and more information about resistance distance we refer to $[11,3,1,10,14,13]$.

We introduce some notation. If $A$ is an $n \times n$ matrix, then for $i, j \in$ $\{1, \ldots, n\}, A(i \mid j)$ will denote the submatrix of $A$ obtained by deleting row $i$ and column $j$. For $i, j \in\{1, \ldots, n\}, i \neq j, A(i, j \mid i, j)$ will denote the principal submatrix of $A$ obtained by deleting rows $i, j$ and columns $i, j$. If $S, T \subset\{1, \ldots, n\}$, we denote by $A[S \mid T]$, the submatrix of $A$ formed by the rows indexed by $S$ and the columns indexed by $T$.

Lemma 1 Let $G$ be a weighted graph with $V(G)=\{1, \ldots, n\}$, and let $L$ be the Laplacian of $G$. Then for $i, j \in\{1, \ldots, n\}, i \neq j$,

$$
r_{i j}=\frac{\operatorname{det} L(i, j \mid i, j)}{\operatorname{det} L(i \mid i)} .
$$

It may be remarked that $\operatorname{det} L(i \mid i)$ equals the number of spanning trees of $G$, by the Matrix-Tree Theorem. 
Let $G$ be a connected graph. A spanning 2-tree of $G$ is defined as a spanning forest of $G$ with two components. A spanning 2-tree is said to separate vertices $i$ and $j$ if the vertices are in distinct components of the 2 -tree. If $G$ is a weighted graph, then the weight of a spanning tree, or a spanning 2-tree, is defined to be the product of the edge weights of the tree, or 2-tree.

Lemma 2 Let $G$ be a weighted graph with $V(G)=\{1, \ldots, n\}$, and let $i, j \in$ $\{1, \ldots, n\}, i \neq j$. Then $r_{i j}$ equals the sum of the weights of all spanning 2 -trees of $G$ separating $i$ and $j$, divided by the sum of the weights of all spanning trees of $G$.

If $A$ is an $m \times n$ matrix, then an $n \times m$ matrix $G$ is said to be a generalized inverse of $A$ if $A G A=A$. For basic properties of generalized inverse see, for example, $[2,6]$.

Lemma 3 Let $G$ be a weighted graph with $V(G)=\{1, \ldots, n\}$, and let $L$ be the Laplacian of $G$. Let $H$ be a generalized inverse of $L$. Then for $i, j \in\{1, \ldots, n\}$,

$$
r_{i j}=h_{i i}+h_{j j}-h_{i j}-h_{j i}
$$

In this paper we consider two specific graphs, the wheel and the fan, and obtain explicit formulas for the resistance distance between any two vertices in these graphs.

\section{Resistances in wheel and fan}

The join $G=G_{1}+G_{2}$ of graphs $G_{1}$ and $G_{2}$ with disjoint vertex sets $V_{1}$ and $V_{2}$, and edge sets $X_{1}$ and $X_{2}$, is the graph union $G_{1} \cup G_{2}$ together with all the edges joining $V_{1}$ and $V_{2}$.

A wheel graph $W_{n}$ is by definition, $K_{1}+C_{n}$, where $C_{n}$ denotes the cycle with $n$ vertices. In the wheel, the vertices corresponding to the cycle $C_{n}$ are labeled from 1 to $n$ in cyclic order, and the central vertex corresponding to $K_{1}$ is labeled as $n+1$. We also consider a weighted wheel in which the edges 
$\{n+1, i\}, i \in\{1,2, \cdots, n\}$, called the spokes of the wheel, have the same weight $\alpha$.

The fan graph $F_{a n}$ is defined as $K_{1}+P_{n}$, where $P_{n}$ is the path on $n$ vertices. All the vertices of the fan corresponding to the path $P_{n}$ are labeled from 1 to $n$ consecutively. The vertex in the fan corresponding to the point $K_{1}$ is labeled $n+1$.

We find the resistance distance between any two vertices in a wheel or a fan in terms of Fibonacci and generalized Fibonacci numbers. We have specifically considered the two classes of graphs together as they are related. The fan is just a wheel with an edge of the cycle removed.

We introduce some notation. Recall that $\alpha$ is the weight of a spoke in a weighted wheel. Let

$$
\gamma=2+\frac{1}{\alpha}, \mu=\frac{\gamma+\sqrt{\gamma^{2}-4}}{2}, \nu=\frac{\gamma-\sqrt{\gamma^{2}-4}}{2} .
$$

We define $G_{k}$ as the generalized Fibonacci number,

$$
G_{k}=\frac{\mu^{k}-\nu^{k}}{\mu-\nu}
$$

Note that when $\alpha=1, G_{k}=F_{2 k}$, where $F_{r}$ is the $r^{t h}$ Fibonacci number. The next result is well-known. We include a proof for completeness.

Lemma 4 Let

$$
M_{n}=\left[\begin{array}{ccccc}
\gamma & -1 & 0 & \cdots & 0 \\
-1 & \gamma & -1 & 0 & \cdots \\
\vdots & & & & \\
0 & \cdots & -1 & \gamma & -1 \\
0 & \cdots & 0 & -1 & \gamma
\end{array}\right]
$$

be $n \times n$. Let $D_{n}=\operatorname{det} M_{n}$. Then $D_{n}=G_{n+1}$.

Proof: Expanding $\operatorname{det} M_{n}$ along the first row, we see that $D_{n}$ satisfies the recurrence relation

$$
D_{n}=D_{n-1}-D_{n-2},
$$


with the initial conditions, $D_{1}=\gamma, D_{2}=\gamma^{2}-1$. Solving the recurrence in the standard fashion, we see that

$$
D_{n}=\delta_{1}\left(\frac{\gamma+\sqrt{\gamma^{2}-4}}{2}\right)^{n}+\delta_{2}\left(\frac{\gamma+\sqrt{\gamma^{2}-4}}{2}\right)^{n}
$$

for some $\delta_{1}$ and $\delta_{2}$. Using the initial conditions it follows that $D_{n}=G_{n+1}$.

Theorem 5 Let $n \geq 3$ be a positive integer. The following results hold for $W_{n}$ :

1. The resistance distance between vertex $n+1$ and vertex $i, i \in$ $\{1,2, \cdots, n\}$, is $r_{n+1, i}=r_{i, n+1}=\frac{G_{n}^{2}}{G_{2 n}-2 G_{n}}$

2. The resistance distance between vertices $i, j \in\{1,2, \cdots, n\}$ is

$$
r_{i j}=\frac{G_{n}^{2}}{G_{2 n}-2 G_{n}}\left[2-\frac{G_{2 k}}{G_{k}}\right]+G_{k},
$$

where

$$
k=\left\{\begin{array}{cl}
|j-i| & \text { if }|j-i| \leq\left\lfloor\frac{n}{2}\right\rfloor . \\
n-|j-i| & \text { if }|j-i|>\left\lfloor\frac{n}{2}\right\rfloor .
\end{array}\right.
$$

Proof: The Laplacian of $W_{n}$ is the $(n+1) \times(n+1)$ matrix

$$
L=\left[\begin{array}{ccccccccc}
\gamma & -1 & 0 & 0 & \cdots & 0 & 0 & -1 & -\frac{1}{\alpha} \\
-1 & \gamma & -1 & 0 & \cdots & 0 & 0 & 0 & -\frac{1}{\alpha} \\
\vdots & & & & & & & & \\
0 & 0 & 0 & \cdots & 0 & -1 & \gamma & -1 & -\frac{1}{\alpha} \\
-1 & 0 & 0 & \cdots & 0 & 0 & -1 & \gamma & -\frac{1}{\alpha} \\
-\frac{1}{\alpha} & -\frac{1}{\alpha} & -\frac{1}{\alpha} & -\frac{1}{\alpha} & \cdots & -\frac{1}{\alpha} & -\frac{1}{\alpha} & -\frac{1}{\alpha} & \frac{n}{\alpha}
\end{array}\right]
$$

Let

$$
H=\left[\begin{array}{cc}
L(n+1 \mid n+1)^{-1} & 0 \\
0 & 0
\end{array}\right] .
$$

Then $H$ is a generalized inverse of $L$. By Lemma 3 , the resistance distance $r_{i j}$ between vertices $i$ and $j$ is given by

$$
r_{i j}=h_{i i}+h_{j j}-h_{i j}-h_{j i} .
$$


Note that

$$
L(n+1 \mid n+1)=\gamma I-P-P^{n-1},
$$

where

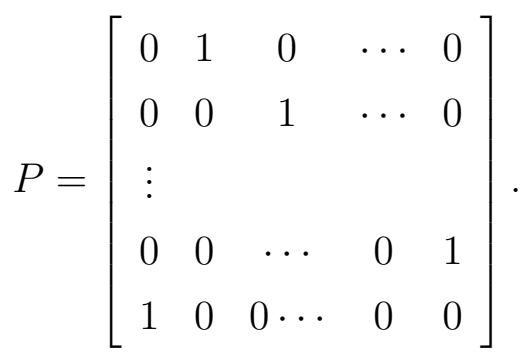

The inverse of $L(n+1 \mid n+1)$ must be a polynomial in $P$ of the following form. If $n=2 m+1$, then

$$
\begin{gathered}
L(n+1 \mid n+1)^{-1}=\beta_{0} I+\beta_{1}\left(P+P^{2 m}\right)+\beta_{2}\left(P^{2}+P^{2 m-1}\right)+\cdots \\
+\beta_{r}\left(P^{r}+P^{2 m+1-r}\right)+\cdots+\beta_{m-1}\left(P^{m-1}+P^{m+2}\right)+\beta_{m}\left(P^{m}+P^{m+1}\right),
\end{gathered}
$$

and if $n=2 m$, then

$$
\begin{aligned}
& L(n+1 \mid n+1)^{-1}=\beta_{0} I+\beta_{1}\left(P+P^{2 m-1}\right)+\beta_{2}\left(P^{2}+P^{2 m-2}\right)+\cdots \\
& \quad+\beta_{r}\left(P^{r}+P^{2 m-r}\right)+\cdots+\beta_{m-1}\left(P^{m-1}+P^{m+1}\right)+\beta_{m}\left(P^{m}\right) .
\end{aligned}
$$

Using $L(n+1 \mid n+1) L(n+1 \mid n+1)^{-1}=I$ and $P^{n}=I$, and comparing the coefficients of $P$, we get,

$$
\gamma \beta_{0}-2 \beta_{1}=1
$$

and

$$
\gamma \beta_{k}-\beta_{k-1}-\beta_{k+1}=0, k \in\{1,2, \cdots, m+1\},
$$

where $\beta_{m+1}=\beta_{m}$ if $n=2 m+1$ and $\beta_{m+1}=\beta_{m-1}$ if $n=2 m$.

Solving (9) as a recurrence relation we get

$$
\beta_{k}=C_{1}\left(\frac{\gamma+\sqrt{\gamma^{2}-4}}{2}\right)^{k}+C_{2}\left(\frac{\gamma-\sqrt{\gamma^{2}-4}}{2}\right)^{k} .
$$

Putting $k=0,1$ in (10) and using (8) we get

$$
\beta_{0}=C_{1}+C_{2}
$$

and

$$
\beta_{1}=\frac{\gamma\left(C_{1}+C_{2}\right)}{2}+\frac{\sqrt{\gamma^{2}-1}\left(C_{1}-C_{2}\right)}{2}
$$


Solving (11) and (12) we get

$$
C_{1}=\frac{1}{\sqrt{\gamma^{2}-4}}\left(\frac{\beta_{0} \sqrt{\gamma^{2}-4}-1}{2}\right), C_{2}=\frac{1}{\sqrt{\gamma^{2}-4}}\left(\frac{\beta_{0} \sqrt{\gamma^{2}-4}+1}{2}\right) .
$$

Hence

$$
\begin{aligned}
\beta_{k} & =\frac{1}{\sqrt{\gamma^{2}-4}}\left[\left(\frac{\beta_{0} \sqrt{\gamma^{2}-4}-1}{2}\right)\left(\frac{\gamma+\sqrt{\gamma^{2}-4}}{2}\right)^{k}+\left(\frac{\beta_{0} \sqrt{\gamma^{2}-4}+1}{2}\right)\left(\frac{\gamma-\sqrt{\gamma^{2}-4}}{2}\right)^{k}\right] \\
& =\frac{1}{\sqrt{\gamma^{2}-4}}\left[\left(\frac{\beta_{0} \sqrt{\gamma^{2}-4}}{2}\left(\mu^{k}+\nu^{k}\right)\right]-\frac{1}{2 \sqrt{\gamma^{2}-4}}\left(\mu^{k}-\nu^{k}\right)\right. \\
& =\frac{\beta_{0}}{2}\left(\mu^{k}+\nu^{k}\right)-\frac{1}{2 \sqrt{\gamma^{2}-4}}\left(\mu^{k}-\nu^{k}\right) \\
& =\frac{\beta_{0}}{2} \frac{G_{2 k}}{G_{k}}-\frac{G_{k}}{2} .
\end{aligned}
$$

It remains to find $\beta_{0}$, and this is achieved using the fact that $\beta_{0}$ is the $(1,1)$-element of $L(n+1 \mid n+1)^{-1}$. Since $\operatorname{det} L(1, n+1 \mid 1, n+1)=D_{n-1}$ and

$$
\operatorname{det} L(n+1 \mid n+1)=\gamma D_{n-1}-2 D_{n-2}-2,
$$

$\beta_{0}=\frac{D_{n-1}}{\operatorname{det}(L(n+1 \mid n+1)}$, which, on simplification gives $\beta_{0}=\frac{G_{n}^{2}}{G_{2 n}-2 G_{n}}$.

Recall from (6), 7 and 2

$$
\begin{gathered}
h_{i, j}=0 \text { if } i=n+1 \text { or } j=n+1 \\
h_{i, j}=\beta_{k} \text { otherwise }
\end{gathered}
$$

where

$$
k=\left\{\begin{array}{cl}
|j-i| & \text { if }|j-i| \leq\left\lfloor\frac{n}{2}\right\rfloor . \\
n-|j-i| & \text { if }|j-i|>\left\lfloor\frac{n}{2}\right\rfloor .
\end{array}\right.
$$

Also from (3)

$$
r_{i j}=h_{i i}+h_{j j}-h_{i j}-h_{j i} .
$$

Clearly, the resistance distance across the spoke $(i, n+1), i \in\{1,2, \cdots, n\}$, is $\beta_{0}$, since $h_{i, n+1}=h_{n+1, i}=0$ for all $i \in\{1,2, \cdots, n\}$. It is also easy to see that the resistance between two vertices $i$ and $j$ on the circumference of the wheel, where the numbers are assigned to vertices in a cyclic order, is $r_{i j}=2\left(\beta_{0}-\beta_{k}\right)$, where

$$
k=\left\{\begin{array}{cl}
|j-i| & \text { if }|j-i| \leq\left\lfloor\frac{n}{2}\right\rfloor . \\
n-|j-i| & \text { if }|j-i|>\left\lfloor\frac{n}{2}\right\rfloor .
\end{array}\right.
$$

This completes the proof. 
Corollary 6 Let $n \geq 3$ be a positive integer and let $W_{n}$ be the unweighted wheel on $n+1$ vertices. Then the following assertions hold:

1. The resistance distance between vertex $n+1$ and vertex $i \in\{1,2, \cdots, n\}$ is $r_{n+1, i}=r_{i, n+1}=\frac{F_{2 n}^{2}}{F_{4 n}-2 F_{2 n}}$

2. The resistance distance between vertices $i, j \in\{1,2, \cdots, n\}$ is

$$
\begin{array}{r}
r_{i j}=\frac{F_{2 n}^{2}}{F_{4 n}-2 F_{2 n}}\left[2-\frac{F_{4 k}}{F_{2 k}}\right]+F_{2 k}, \\
\text { where } k=\left\{\begin{array}{cl}
|j-i| & \text { if }|j-i| \leq\left\lfloor\frac{n}{2}\right\rfloor \\
n-|j-i| & \text { if }|j-i|>\left\lfloor\frac{n}{2}\right\rfloor
\end{array}\right.
\end{array}
$$

and where $F_{k}$ is the $k^{\text {th }}$ Fibonacci number.

Proof: Clearly $G_{k}=F_{2 k}$ when $\alpha=1$ i.e., $\gamma=2+\frac{1}{\alpha}=3$. The result follows from Theorem 5 .

We now turn to the fan graph $F a n_{n}$ defined earlier.

Theorem 7 Let $n \geq 1$ be a positive integer. Then for the fan graph Fan , the following results hold:

1. The resistance distance between vertex $n+1$ and vertex $i \in\{1,2, \cdots, n\}$ is $\frac{F_{2(n-i)+1} F_{2 i-1}}{F_{2 n}}$.

2. The resistance distance between vertices $i, j \in\{1,2, \cdots, n\}, i<j$ is

$$
\frac{F_{2(n-j)+1}\left(F_{2 j-1}-F_{2 i-1}\right)+F_{2 i-1}\left(F_{2(n-i)+1}-F_{2(n-j)+1}\right)}{F_{2 n}} .
$$

Proof: The result can be proved either by evaluating determinants and using Lemma 1, or by combinatorial arguments. We prove the first statement by evaluating determinants. 
The Laplacian of Fan $_{n}$ is the $(n+1) \times(n+1)$ matrix

$$
L=\left[\begin{array}{ccccccccc}
2 & -1 & 0 & 0 & \cdots & 0 & 0 & 0 & -1 \\
-1 & 3 & -1 & 0 & \cdots & 0 & 0 & 0 & -1 \\
\vdots & & & & & & & & \\
0 & 0 & 0 & \cdots & 0 & -1 & 3 & -1 & -1 \\
0 & 0 & 0 & \cdots & 0 & 0 & -1 & 2 & -1 \\
-1 & -1 & -1 & -1 & \cdots & -1 & -1 & -1 & n+1
\end{array}\right]
$$

By Lemma 1,

$$
r_{n+1,1}=\frac{\operatorname{det} L(n+1,1 \mid n+1,1)}{\operatorname{det} L(n+1 \mid n+1)}
$$

We continue to use the notation $D_{n}$ introduced in Lemma 4 . However we keep in mind that now $\gamma=3$.

By Lemma 4,

$$
\operatorname{det} L(n+1 \mid n+1)=F_{2 n} .
$$

Thus the number of spanning trees in $\mathrm{Fan}_{n}$ is $F_{2 n}$. This result is in fact wellknown (see [8], p.362).

Using Laplace expansion and Lemma 4 we get

$$
\begin{aligned}
\operatorname{det} L(n+1,1 \mid n+1,1) & =2 \operatorname{det} L(n+1, n, 1 \mid n+1, n, 1) \\
& -\operatorname{det} L(n+1, n, 1, n-1 \mid n+1,1, n, n-1) \\
& =2 D_{n-2}-D_{n-3} \\
& =2 F_{2 n-2}-F_{2 n-4} \\
& =F_{2 n-2}+F_{2 n-3}+F_{2 n-4}-F_{2 n-4} \\
& =F_{2 n-1} .
\end{aligned}
$$

Hence $r_{n+1,1}=\frac{F_{2 n-1}}{F_{2 n}}$. It can be similarly shown that

$$
\begin{aligned}
\operatorname{det}(L(n+1, i \mid n+1, i) & =\operatorname{det} L[1, \cdots, i-1 \mid 1, \cdots, i-1] \\
& \times \operatorname{det} L[i+1, \cdots, n \mid i+1, \cdots, n] \\
& =F_{2 i-1} \times F_{2(n-i+1)-1}
\end{aligned}
$$

Therefore the first statement is true. 
We shall give a combinatorial proof of the second statement. Let $i, j \in$ $\{1, \ldots, n\}, i<j$ be fixed. We must calculate the number of spanning 2 -trees separating $i$ and $j$. First consider the number of spanning 2-trees with components $T_{i}$ and $T_{j}$, where $T_{i}$ contains $i$ and $n+1$. Then $T_{j}$ must be a path containing $j$. Let $i \leq k \leq j-1$ be the vertex between $i$ and $j$ such that $k$ is in $T_{i}$ and $k+1$ is in $T_{j}$. Let $j \leq \ell \leq n$ be the vertex between $j$ and $n$ such that $\ell-1$ is in $T_{j}$ and $\ell$ in tree $T_{i}$. Then $T_{i}$ consists of a spanning tree of the fan with vertices $1,2 \cdots k$ and $n+1$, along with a spanning tree of the fan with vertices $\ell, \cdots, n$ and $n+1$ or none of these vertices. Since the number of spanning trees in a fan with $m+1$ vertices is $F_{2 m}$, therefore the number ways in which $T_{i}$ can be selected is

$$
\left(\sum_{k=i}^{j-1} F_{2 k}\right)\left(\left(\sum_{l=j+1}^{n} F_{2(n-l+1)}\right)+1\right) .
$$

Now let us find the number of spanning 2-trees with two components such that $i$ and $j$ are in the two components $T_{i}$ and $T_{j}$ respectively, and $n+1$ is in $T_{j}$. This case is similar to the previous case. Relabel the fan $F_{a n}$ such that the vertex corresponding to $K_{1}$ remains labeled as $n+1$, but the vertex $i$ in $\operatorname{Fan}_{n}$ corresponding to the $i^{\text {th }}$ vertex in the path $P_{n}$ is labeled as $n-i+1$. In this new labeling, the vertex formerly labeled $j$ is labeled $n-j+1$, and vertex formerly labeled $i$ is labeled $n-i+1$. Thus $n-j+1<n-i+1$. Using similar technique as before, we get the number of spanning 2-trees such that $i$ is in one components, and $j$ and $n+1$ are in the other component as

$$
\left(\sum_{k=n-j+1}^{n-i} F_{2 k}\right)\left(\left(\sum_{l=n-i+2}^{n} F_{2(n-l+1)}\right)+1\right)
$$

Hence the total number of spanning 2-trees of $F a n_{n}$ with separating $i$ and $j$ is

$$
\left(\sum_{k=i}^{j-1} F_{2 k}\right)\left(\left(\sum_{l=j+1}^{n} F_{2(n-l+1)}\right)+1\right)+\left(\sum_{k=n-j+1}^{n-i} F_{2 k}\right)\left(\left(\sum_{l=n-i+2}^{n} F_{2(n-l+1)}\right)+1\right),
$$

By using the fact that $\sum_{i=1}^{r} F_{2 i}=F_{2 i+1}-1$, The above expression reduces to:

$$
F_{2(n-j)+1} \times\left(F_{2 j-1}-F_{2 i-1}\right)+F_{2 i-1} \times\left(F_{2(n-i)+1}-F_{2(n-j)+1}\right) .
$$




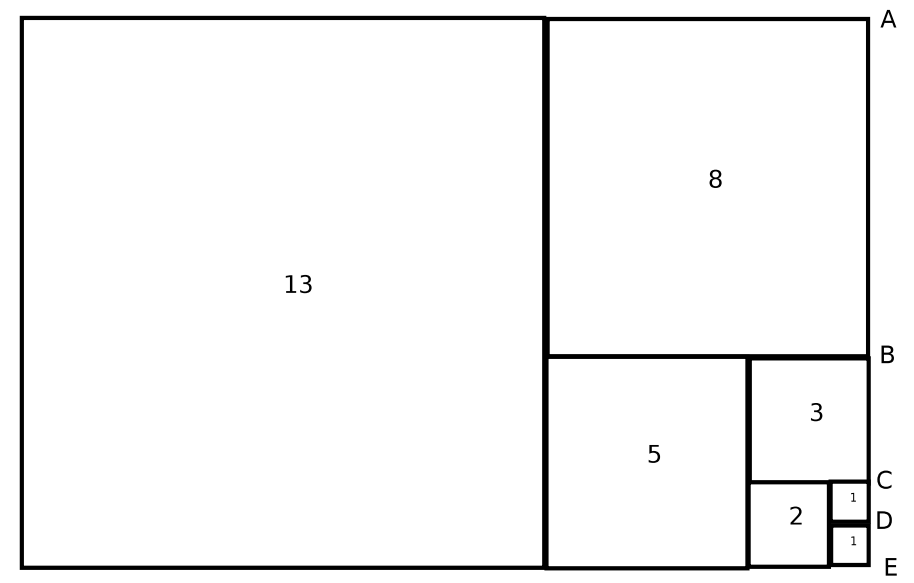

Figure 1: Fibonacci tiling of a rectangle with sides $13 \times 21$

Dividing (15) by $F_{2 n}$, the number of spanning forests in $F a n_{n}$, (ii) is proved.

\section{Fibonacci Tilings}

We describe as Fibonacci Tiling as a tiling of a rectangle of side $F_{n}$ and $F_{n-1}$ with $n-1$ squares of lengths $F_{1}, F_{2}, \cdots, F_{n-1}$. This tiling has a very interesting relation with the squaring a rectangle problem and Fan graphs. Squaring a rectangle problem as described by Brooks et. al. [5] is the problem of dividing a rectangle into a finite number of non-overlapping squares, no two of which are equal. Note the Fibonacci tiling does not exactly fit into this description since two squares have same lengths namely $F_{1}$ and $F_{2}$, but this defect can be easily corrected by tiling one of the squares with another known tiling for a square (To know more see[9]).

Now let us describe the relation of Fan graphs to Fibonacci tilings. We apply the general approach for converting a squared rectangle to an electrical network as described in [3]. Consider the squared rectangle shown in Fig. 1. Let us cut this rectangle out of a sheet of nichrome or any other material with low conductivity and let us put rods made of silver or some material with high 


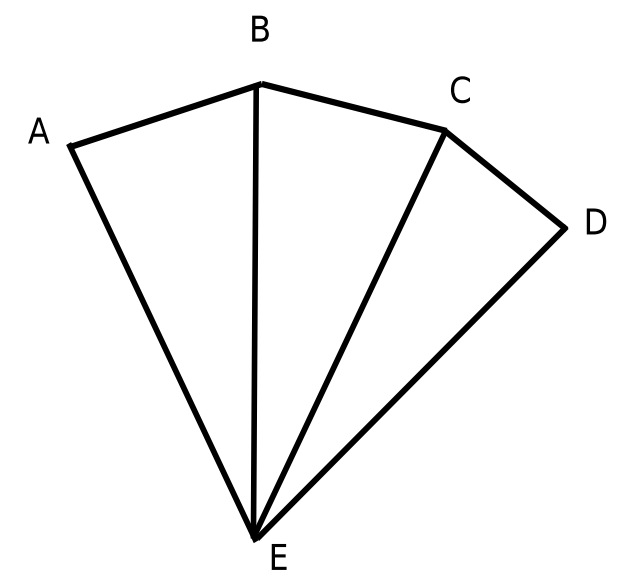

Figure 2: Electrical network corresponding to the Fibonacci tiling in Fig. 1

conductivity at the top and bottom. Now if we ensure that the silver rod at the top is at 13 volts and the rod at the bottom is kept at 0 volts, the current will flow from top to bottom and the potential at a point at height $x$ will be $x$. Since no current flows horizontally across the rectangle, the current will not change if (1) we place silver rods on the horizontal sides of the squares and (2) cut narrow slits along the vertical sides.

Now since silver is a very good conductor, the points of each silver rod have been shortened, so can be identified (as A,B,C,D,E in Fig. 1). Thus as an electric conductor, the whole rectangle behaves like the Fan shown in Fig. 2. Now it is clear that the resistance distance between node A and E in Fig. 2 is nothing but the resistance of the rectangular conductor between rods $\mathrm{A}$ and $\mathrm{E}$ in Fig. 1 i.e., $\frac{13}{21}$. Also, it is interesting to note that Fibonacci tiling gives a geometrical proof of the sum of Fibonacci numbers, sum of odd/even indexed Fibonacci numbers and sum of squares of Fibonacci numbers. 


\section{References}

1. R.B. Bapat, Resistance distance in graphs, Mathematics Student, Vol.68, Nos.1-4(1999) 87-98

2. Adi Ben-Israel and Thomas N.E. Greville, Generalized Inverses. Theory and Applications, Second ed., Springer, New York, 2003

3. Belá Bollobás, Modern Graph Theory, Springer-Verlag, New York, 1998.

4. J.A. Bondy and U.S.R. Murty, Graph Theory, Graduate Texts in Mathematics, 244, Springer, New York, 2008

5. R. L. Brooks, C. A. B. Smith, A. H. Stone, W. T. Tutte, The dissection of rectangles into squares, Duke Math Journal, 7, 312-40.

6. S.L. Campbell and C.D. Meyer, Generalized Inverses of Linear Transformation, Pitman, 1979

7. Chris Godsil and Gordon Royle,Algebraic Graph Theory, Springer, 2001.

8. R. L. Graham, D. E. Knuth, O. Patashnik, Concrete Mathematics, Second edition, Pearson Education (2007).

9. Frederick V. Henle and James M. Henle, Squaring the Plane, American Mathematical Monthly, Volume 115, Number 1, January 2008 , pp. 3$12(10)$.

10. D.J. Klien, Resistance-distance sum rules, Croatica Chemica Acta $75(2)(2002)$ 633-649

11. D.J. Klein and M. Randić, Resistance distance, Journal of Mathematical Chemistry, 12(1993) 81-95

12. W. T. Tutte, A theory of 3-connected graphs, Nederl. Akad. Wentensch. Proc. Ser. A, 23, 441-55

13. Wenjun Xiao, Ivan Gutman, On resistance matrices. MATCH Commun. Math. Comput. Chem. No. 49, (2003), 67-81. 
14. Wenjun Xiao, Ivan Gutman, Relations between resistance and Laplacian matrices and their applications. MATCH Commun. Math. Comput. Chem. No. 51, (2004), 119-127. 